\title{
Organizational and pedagogical basis for the development of science and education networks
}

\author{
Natalia N. Davydova ${ }^{1, *}$, Evgeny M. Dorozhkin ${ }^{1}$, and Vladimir A. Fedorov ${ }^{1}$ \\ ${ }^{1}$ Russian State Vocational Pedagogical University, 620012, Yekaterinburg, Russia
}

\begin{abstract}
In the new model of education, the training is diverse and variable, focused on new educational results. This requires the development of corporate processes and stimulates the expansion of cooperation of open educational systems in the conditions of network interaction. The paper presents approaches to determining the content and conditions of a new direction of development of network interaction of educational organizations in the form of scientific and educational networks. It is shown that the main purpose of the research in the conditions of the scientific and educational network is to identify new knowledge about pedagogical processes in specific organizations - participants of interaction, their distinctive features, objective natural connections between pedagogical processes and phenomena.
\end{abstract}

\section{Introduction.}

Under the conditions of the new economic structure and social modernization, there is a need for a person who is capable of creative work, self-development, search and introduction of education, the forms and methods of its organization of new professional knowledge, collective and independent activities. Therefore, only by transforming the content, the education system can become a resource for the development of the modern economy. In the national projects for the period up to 2024 , the acceleration of the transition to the path of innovative development is indicated as the main strategic task to change the socio-economic system of the country [1]. The effectiveness of these changes depends on the development of education, which creates the main resource of the country's development - human potential. To solve this problem, we need systemic changes associated with the formation of real readiness for innovation in educational systems. It should be noted that the full-scale innovative activity, capable of ensuring the evolutionary transition of educational organizations to the updated effective practices, in recent years is increasingly associated with the use of a network approach [2]. At the same time, in the practice of development of network interaction in education, ensuring its effectiveness and efficiency, there are a number of problems, the solution of which is associated with the development of appropriate theoretical and methodological support.

\section{Literature review}

Speaking about the use of the concept of "network" in modern social knowledge, it should be noted that it has many dimensions and levels of reflection. Network theory, network approach, network communities, network culture and virtual social networks, and interfirm organizational networks of knowledge etc. are being discussed. The main features of knowledge networks were multidimensional and multi-layered, a variety of methodological principles of their development $[3,4]$. To a significant extent, this is due to the significant popularity of this subject, which contributes to the fact that knowledge about networks simultaneously "grows" in various sciences and fields of knowledge - cognitive science, sociology, economics, pedagogy, politics, management, semiotics, etc. $[5,6]$. At the same time, today there is a growing desire to formalize knowledge about networks, to create a unified theory of networks with all the supposed attributes of strict scientific knowledge [6]. Consequently, the multidimensionality and diversity of ideas about networks in modern the "network" are not so much a concept as a kind social cognition actualizes questions not only about the term "network", but also about the prospects of development of this research area $[7,8$, and 9]. All of the above suggests that umbrella productive metaphor combines a gestalt, perspective consideration of the social world $[10,11]$. The metaphor of the network is used frequently and productively. The very image of the network reflects the spatial unevenness of the fabric of social life, as well as the dynamics (and the very possibility) of its transformation (the ability to "weave the network"). At the same time, it is important to emphasize that network configurations are extremely dynamic - the center and periphery can change, or rather, "move" through the network. It should be understood that the art of creating a network is the art of connecting and holding together the heterogeneous elements included. Among the general principles that

\footnotetext{
* Corresponding author: edscience@mail.ru
} 
apply to all types of networks, we can distinguish the following:

- networks are changing, evolving over time structures;

- heterogeneous networks. Only a very small number of central elements attract the mass of the rest. Among the others, most items are related to only a few of the closest;

- the distance between the network elements is small. It is much smaller than if the network was created randomly. On the social network, all people know each other through an average of six intermediate handshakes. In the "world wide web" the path between any two documents is an average of 14 mouse clicks;

- there is a systemic effect in networks: new properties appear in the network, which its subsystems did not have, i.e. networks are characterized by emergence;

- for heterogeneous systems, which include groups of people and software, Pareto's law applies: $20 \%$ of efforts give $80 \%$ of the result, and the remaining $80 \%$ of efforts - only $20 \%$ of the result.

Describing the term "network interaction", it can be noted that it is one of the most frequently used in cases when it comes to establishing relationships between different social systems or their individual components [7 - 9]. The main features of network interaction are coordination, the lack of a single center, a strictly centralized management structure, the predominance of self-organization structures. It should also be noted that the boundaries of the network in this case are not set, the rules of behavior of the elements are mainly informal, and the networks themselves are most often created for the development and integration of efforts in the performance of large tasks. Moreover, it is checked by practice that the network exists only as long as it gives to each participant more, than he gives it.

The analysis of the functioning of networks conducted by the authors showed that their development took place in the conditions of advancing the theory by practice. Therefore, the purpose of this work is to determine the content and conditions of a new direction of development of network interaction in the form of scientific and educational networks.

\section{Research methodology.}

To substantiate the complex structure, content and conditions of network interaction in education, where there are both integration trends and deep, not clearly expressed, elusive processes, the activity approach was used. It allows one to assess the state of the scientific and educational network, to identify possible options for its development, and socio-cultural approach, which is used in the analysis of educational networks as socio-cultural situations, determining the social and cultural significance of network forms of co-organization in the education system.

\section{Results and discussion.}

To fully understand the nature of scientific and educational networks, it is necessary to identify the conditions that determined their creation and development. One of the important conditions for the emergence of network interactions is integration processes that occur in the education system in different forms and at different levels [12, 13].

It is integration that has two major advantages: first the ability to enhance specialization and, at the same time, the integration of the vital functions of the merging components and thereby increase the viability of the system as a whole. The second advantage is the possibility of cross-compensation for resource losses or deficits through the establishment of an effective system of communication and exchange.

The desire of educational organizations for selfdevelopment and self-organization also contributes to the creation and development of scientific and educational networks. It is known that the emergence of the new is possible where appropriate opportunities are provided for this, where there is greater freedom of choice. In particular, in education, it is the excessive diversity that creates a range of possible directions for the development of specific educational systems, provides material for the selection of optimal trends in this development. In order for education to be capable of social construction, cultural creation, and not only the translation of knowledge and norms, the processes of development of educational systems should encourage educational organizations to self-development and selforganization.

Let us note that if self-development characterizes the target process of changing the object in time under the influence of its internal, immanent causes (causa sui), then self-organization characterizes the structural mechanism, the complexity of the internal structure of the object due to the interactions between its elements $[14,15]$. The prefix "self" - (self, selbst) characterizes the attribute characteristics of the existence of an object, not its material properties. This is the understanding of the categories of "self-development" and "selforganization" from Aristotle to A.I. Prigogine [16].

It can be stated that self-organization is the establishment of organization, order due to the coordinated interaction of components within the system in the absence of ordering effects from the environment.

For a system to be self-organizing and therefore able to evolve progressively, it must meet at least the following requirements:

- be open, i.e. exchange matter, energy or information with the environment;

- the processes taking place in it should be cooperative (corporate), i.e. the actions of its components should be consistent with each other;

- be dynamic, stay away from balance.

Therefore, in the most general form, network interaction can be defined as a collective distributed activity, including a set of relations between all subjects of innovative development. At the same time, knowledge and information become the main resource of production 
in the community of network structures, and those who have knowledge become the dominant social group.

The concept of "scientific and educational network" has a special meaning. On the one hand, the network acts as a set of established interests of creative teams representing various educational organizations engaged in the development of new scientific knowledge (scientific component). It is known that the difference between scientific knowledge and any other is that scientific knowledge is justified and reveals a set of stable features of the object of study, defines the subject in terms of belonging to any category, highlighting the criteria and principles inherent in all phenomena and subjects of the category. Scientific knowledge is systematically organized and has its own language, which is based on the categorical apparatus of science, in our case, pedagogy.

Chris Collinson and Jeff Purcell noted that knowledge within the network community was distributed evenly; working methods are created and applied together, which methods function better than others, how and when it is more appropriate to apply them. Such communities "are in the service of knowledge and competencies in a particular type of activity of the organization" [17], help participants to develop their own professionalism, make valuable contributions to the overall work. Scientific research, leading to scientific knowledge, is always a special process, which consists of the cognitive activity of people, means of knowledge, its objects and knowledge. The specificity of scientific knowledge is that cognitive activity in science is carried out not by all, but by specially trained groups of people, and the form of its development becomes scientific research. Therefore, the basis for the development of subjects of network interaction within the framework of the scientific and educational network is the movement to knowledge in the selected field of knowledge based on modeling and experimentation. In this regard, the main purpose of pedagogical research in the conditions of scientific and educational network is to identify new knowledge about the pedagogical processes in specific organizations participants of interaction, their distinctive features (structure, action, history of development), objective natural connections between pedagogical processes and phenomena. It is the network that allows to convert (replace, modify) knowledge, for example, to combine and recombine knowledge and experience, turning them into means of innovation.

At the same time, it is the desire to develop new knowledge about pedagogical processes and phenomena that explains the presence of an educational component in the scientific and educational network aimed at improving the level of methodological culture of participants in network interaction. The educational component is a theoretical basis for the design and construction of the educational process, awareness, formulation and creative solution of pedagogical problems and methodological reflection. These are implemented by full-time distant training of participants in the interaction of theoretical and methodological foundations of innovation in the context of developing cooperation and integration of network entities on the principles of self-development and self-education.

The need to form a methodological culture of working in the network interaction of pedagogical collectives is determined by the current socio-cultural situation, when rapidly and intensively changing modern information society requires rapid adjustment of existing and development of fundamentally new educational programs. Mastering the methodological culture allows the participants of interaction to effectively develop new areas of theory and practice of education, while developing independence in obtaining new knowledge through the formulation and solution of scientific, methodological and creative tasks in this area [2].

Scientific and educational network as a complex system is not reduced to a simple set of elements, and dividing it into separate parts, it is impossible to know all the properties of the system as a whole. Such network is a set of elements (objects, subjects) that are in a certain relationship with each other and make up a unity (integrity), aimed at achieving a certain goal. Research and education network can be part of a system of higher order (super-system) and include a system of lower order (sub). Thus, the concepts of "element", "subsystem", "system", and "super-system" are mutually transformable: the system can be considered as an element of a higher order system, and the element - as a system (in-depth analysis).

Thus, the integrity of the scientific and educational network means the fundamental irreducibility of its properties to the sum of the properties of its constituent elements.

The structure of the scientific and educational network refers to the possibility of describing the network through the establishment of its structure, i.e. network connections and relationships, as well as the conditionality of the behavior of the scientific and educational network is not so much the behavior of its individual elements, as the properties of its structure.

The principle of interdependence of the scientific and educational network and the environment means that the properties of the network are formed and manifested in the process of its interaction with the environment.

The hierarchy of the scientific and educational network characterizes its position in a number of systems: each studied system can be considered as an element of a wider system (super-system, macrosystem); each element of the studied system can be considered as an independent system (Microsystem).

The multi-aspect nature of the scientific and educational network means its complexity. Since any system can be studied from different points of view, for a deep knowledge of the scientific and educational network it is necessary to build a set of models, each of which would describe its specific aspect.

One of the most important for the study of the properties of scientific and educational networks, as well as any other, is their ability to develop.

Thus, according to the authors, the "scientific and educational network" can be considered a dynamic set of interrelated agents, representing scientific, educational, social, cultural organizations (their units, creative 
teams), as well as elements of innovative infrastructure. They are interested in the development of the education system industrial enterprises that operate as a virtual organization and carry out innovative projects in the field of education at a high level of coordination of goals and integration of all types of resources. This is achieved through the formation of the internal information space of the network and resulting in the creation of collective intellectual property in education and increase the external (external) effect. An important effect of the development of the scientific and educational network is the emergence of a network community in which network interaction is carried out, ensuring the achievement of educational goals and the realization of the needs of the individual and society in improving the efficiency and quality of education.

\section{Conclusion.}

Thus, in the new model of education, focused on the development of the educational system of the country as a whole, training becomes diverse and variable, focused on new educational results, which requires the development of corporate processes and stimulates the transition to the expansion of cooperation of open educational systems in a network interaction. In this regard, the main purpose of pedagogical research in the conditions of the scientific and educational network is to identify new knowledge about the pedagogical processes in specific organizations participating in the interaction. At the same time, it is the desire to develop new knowledge about pedagogical processes and phenomena that explains the presence of an educational component in the scientific and educational network aimed at improving the level of methodological culture of participants in network interaction. Taking into account these arguments, it is legitimate to attribute the new concept of "scientific and educational network" to the key in the theory of development of network interaction in education.

\section{References}

1. G. Y. Peshkova, A. Y. Samarina. Digital economy and recruitment potential: strategic interconnection and prospects. The Education and science journal. 20 (10) (2018). https://doi.org/10.17853/1994-56392018-10-50-75

2. Davydova N. N., Dorozhkin E. M. \& Fedorov V. A. Educational research networks principles of organization.IJET,7(2.13)(2018).DOI:

10.14419/ijet.v7i2.13.11573

https://www.sciencepubco.com/index.php/ijet/article/ view/11573
3. M. Remorenko Different management for different education. (St. Petersburg.AEC, 2005).

4. A. I. Adamsky. Network partnership in education. (M: Eureka, 2004)

5. D. A. Novikov, N. P. Glotova. Models and mechanisms of management of educational networks and complexes. (M.: M of RWE., 2004).

6. O. M. Gyshchina, O. P. Mikheeva. Massive open online courses for pedagogical staff training. The Education and science journal. 19(7) (2017). https://doi.org/10.17853/1994-5639-2017-7-119-136

7. Yu. V. Gromyko. Design and programming of education development. (M.: MARO, 1996)

8. R Grant, C. Baden-Fuller. The knowledge-based view of strategic alliance formation: Knowledge accessing versus organizational learning. Cooperative Strategies and Alliances. (Amsterdam: Elsevier Science Ltd., 2003)

9. J. Ziman. "Postacademic science": Constructing knowledge with networks and norms. SS, 9.(1) (1996)

10.9. N. N Davydova, E. M. Dorozhkin, \& V. A Fedorov. Objects And Managing Model Of Development Of Research And Education Networks. EpSBS. $\quad \mathbf{X X X V , \quad 3 1 . ~ ( 2 0 1 8 ) . ~ D O I : ~}$ http://dx.doi.org/10.15405/epsbs.2018.02.31

11. B. Latour. Reassembling the Social. An Introduction to Actor-Network-Theory. (Oxford: Oxford Univ. Press., 2005)

12.E. Wenger. Communities of Practice: learning, meaning and identity. (Cambridge: Cambridge Univ. Press., 1998)

13. N. A. Astashova, S. K. Bondyreva, A. P. Smantser. Development of the axiosphere of the future teacher in the dialogue space of modern education. The Education and science journal. 20(7). (2018). https://doi.org/10.17853/1994-5639-2018-7-32-67

14. Usol'tsev A. P. Inflation of the competition-based approach in the Russian pedagogical science and practical teaching. The Education and science journal.19(1)(2017). https://doi.org/10.17853/19945639-2017-1-9-25

15. S. V. Komarov, S. I. Cordon. Fundamentals of methodology: a system-based approach. (Perm: Publishing house in Perm. State Un., 2005).

16. A. I. Prigozhin. Goals and values. New ways of working with the future. (M.: Academy of national economy, 2010)

17. K. Collinson, D. Purcell; Learn to fly. Practical lessons on knowledge management from the best learning organizations (M.: ICSR, 2006) 